\title{
A Complete Color Normalization Approach and Classification of Breast Cancer Cell
}

\author{
Athira M. A. \\ PG Scholar \\ Dept. of ECE \\ CCET VALANCHERY
}

\author{
Aswathy M. A. \\ Research scholar \\ School of Electronics \& \\ Engineering \\ VIT University Chennai
}

\author{
Nahan Rahman \\ PG Scholar \\ Dept. of CSE \\ CCET VALANCHERY
}

\begin{abstract}
Breast Cancer is one of the severe diseases causing large number of deaths in women. So there is a need for efficient technique that diagnoses such cells without the involvement of human, with high accuracies. In the first phase, a complete normalization scheme is introduced to address the problem of color variation in images jointly caused by inconsistent biopsy staining and non-standard imaging condition. This scheme is robust to parameters and insensitive to content of the image and achromatic colors. Then In second phase, a Feed forward back propagation neural network classify benign and malignant tumor and also classify breast cancer tumor in type1, type 2 and type3. It can be concluded that it works as promising tool for classification of cancer cells. Breast Cancer is one of the severe disease causing large number of deaths in women. So there is a need for efficient technique that diagnoses such cells without the involvement of human, with high accuracies. In the first phase, a complete normalization scheme is introduced to address the problem of color variation in images jointly caused by inconsistent biopsy staining and non-standard imaging condition. This scheme is robust to parameters and insensitive to content of the image and achromatic colors. Then In second phase, a Feed forward back propagation neural network classify benign and malignant tumor and also classify breast cancer tumor in type1, type 2 and type3. It can be concluded that it works as promising tool for classification of cancer cells.
\end{abstract}

\section{Keywords}

Neural Network, Normalizaion, Histopathology and Breast Cancer.

\section{INTRODUCTION}

The out of manage improvement of cells in an organ is called tumors that may be cancerous. There are two kinds of tumors, benign and malignant. Benign or non-cancerous tumors are not spreading and aren't life intimidating. within the other hand, malignant or cancerous tumor are expanding and lifestyles threatening [1]. Malignant breast most cancers is described while the developing cells are within the breast tissue. Breast most cancers is the second one overall purpose of mortality among girls and the first reason of lifeless among them between 40 and 55 a long time [2]. Everyday breast cancer prognosis accompanied by appropriate cancer treatment can lessen the unwilling chance. it's miles counseled to do tumor evaluation check every 4-6 weeks. primarily based on that motive, benign and malignant detection based on class capabilities turn out to be very vital [3].The range of studies works carried out in the vicinity of breast most cancers detection and class. Many university, studies facilities and business institutions are focused on this trouble due to the reality that breast most cancers is becoming the most common form of cancer ailment of today's lady population. thus, the development of a completely automatic most cancers detection machine supporting a human expert has turn out to be a hard and tough assignment $[2,3]$. in recent times many camera based automated breast cancer detection systems need to face the problem of cells and their nuclei separation from the relaxation of the photograph content. so right here we should developed a device for the classification of cancer cells. the principle objective is to hit upon the cancer in early level. and it's far difficult to come across in its early levels due to the fact signs appear only at advanced levels inflicting the mortality rate to be the best among all different styles of most cancers, Breast cancer is the second purpose of useless amongst girls. Early detection accompanied through appropriate most cancers remedy can lessen the lethal danger. medical experts could make errors while identifying a ailment. however, computerized choice aid systems are fraught with troubles that stem from color variations in tissue look. So right here introduces a complete normalization scheme to deal with the problem of color variation in his topathology pics.

\subsection{Contribution}

Right here in these paintings we effectively get rid of the colour versions in histopathology snap shots. In $2 \mathrm{~d}$ section, it is prolonged for the analysis and analysis of most cancers detection. that is it's miles extended to the breast cancer detection and classification of benign and malignant breast tumor primarily based on H E stained histopathology and feed forward again propagation neural community.

\section{2 . Scope}

With the use of equal method some other kind of cancer also can be used to stumble on in predominant programs of cancer detection, At gift the image processing is carried on within the grayscale image, destiny upgrades may be immediately on the color pix. The million order dataset may be selected and photograph class may be executed on large dataset. With accelerated length of dataset numerous troubles inclusive of importing information, managing characteristic set, multiplied execution time of category algorithms and many others. may be taken into consideration. Greater image capabilities can be extracted for better type. Diverse combos of preexisting functions may be used to properly classify scientific information. 


\section{LITERATURE SURVEY}

We categorize histo- pathology image centered colour normalization answers in literature into 3 distinct businesses. the first institution of normalization algorithms are based totally on histogram matching within the RGB color area. In [8], after image history elimination, histogram matching is carried out within the crimson, green, and blue channels, respectively. In [9], colour map quantile matching, a variation of histogram matching, turned into proposed for stable coloration normalization. Considering histogram matching on whole images ignores nearby differences of picture content material, color related to one stain can be matched to beside the point hues. lately, tissue aspect segmentation

followed through histogram landmark matching changed into proposed to take away stain version [14]. As histogram matching based methods do no longer distinguish causes of coloration variant, histological facts is hardly ever preserved after normalization, therefore introducing undesirable bias into subsequent image evaluation.

\subsection{Histogram Matching}

The first institution of normalization algorithms are based on histogram matching in the RGB coloration area. In [8], after picture background elimination, histogram matching is executed within the purple, green, and blue channels, respectively. In [9], color map quantile matching, a version of histogram matching, became proposed for solid coloration normalization. Considering that histogram matching on complete images ignores neighborhood differences of photo content, shade related to one stain may be matched to beside the point colorings. Recently, tissue aspect segmentation observed through histogram landmark matching becomes proposed to take away stain version [14]. As histogram matching based strategies do now not distinguish causes of colour version, histological data is hardly preserved after normalization, therefore introducing unwanted bias into subsequent image evaluation.

\subsection{Color Transfer}

The second one institution of coloration normalization answers is based on the so-referred to as color switch approach mentioned in [15]. After converted to the 1 color area [16], the imply and variance in each shade channel of a query image are matched to the facts of a reference photograph [10]. Considering the fact that snap shots stained by way of more than one chemical dyes may additionally have special shade distributions, colorations related to extraordinary histological components may smear each different after color transfer. To address this problem, earlier than colour transfer, a picture is split into regions by way of segmentation manually [17] or mechanically thru pixel type [11], so that every area carries one kind of histological objects best. For one element, guide segmentation of pix in large datasets is infeasible; For another, segmentation achieved by means of tough pixel classification isn't always dependable because one pixel in a histo-pathology photograph may belong to a couple of items due to histological aspect overlap. subsequently, smooth pixel classification by way of stain decomposition turned into proposed to precede color switch [11], [18]. An advanced normalization method primarily based on work [11] became proposed in [12]. in this technique, mean and ranking data in each decomposition channel of a test picture are mapped to the facts of a reference photograph nonlinearly. however, applying color switch on decomposition consequences has boundaries. First, assets of colour variant in histo-pathology pics are lumped together and now not addressed one by one. second, facts in decomposition channels, which is carefully related to histological statistics in pictures, is changed and as a consequence tissue functions may not be preserved after normalization.

\subsection{Spectral Matching}

This class of shade normalization solutions targets to dispose of stains variant most effective. To that stop, algorithms belonging to this group first estimate stain spectra both the usage of adaptive estimators [7], [19] or via devoted hardware [20], [13], and then in shape the anticipated amount to a reference stain spectra. Procedures on this class have an advantage over other organizations inside the feel that if stain variation is the simplest supply of color disagreements in pix, with appropriate estimation, spectral matching methods can maintain histological functions. How ever, if other causes additionally contribute, as algorithms in this class do not become aware of their consequences, histological functions can be changed after normalization.

\section{PROPOSED SYSTEM}

The block diagram of the proposed approach consisting of two processes is shown in Fig. 1. Here first part is color normalization and other part is training using ANN.

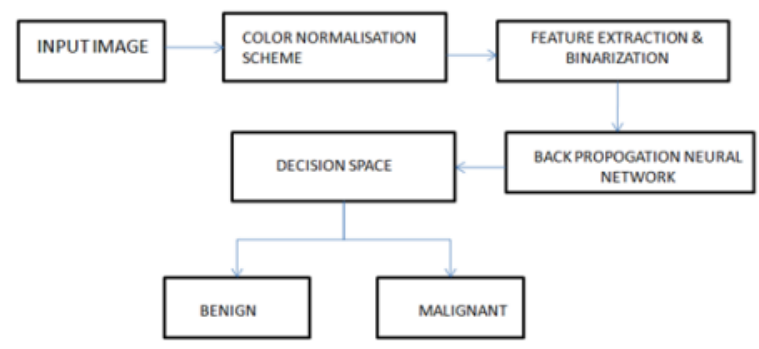

Fig 1 : Block diagram of proposed topology

\subsection{Color Normalisation}

The block diagram of the proposed normalization method including two techniques is shown in Fig. 2:

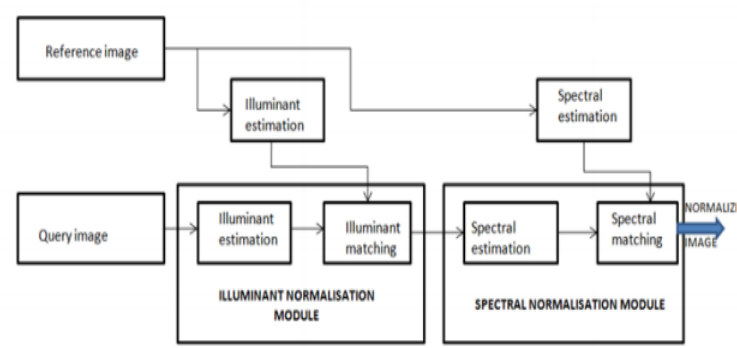

Fig. 2: Diagram of the proposed normalization pipeline, where the on-line normalization processing includes an illuminant normalization module and a stain spectral normalization module.

The off-line system defines a fashionable histo-pathology photograph coaching condition inclusive of records on and , in which the subscript $\mathrm{s}$ labels a trendy condition. For operational flexibility, statistics on the standard situation may 
be described via either predetermined quantities explicitly, or a reference photograph as a reduced set implicitly. in the diagram, a reference photo Is is used, wherein a well known imaging illuminant and stain spectra are obtained by illuminant estimation and spectral estimation, respectively. notice, to qualify an image to be a reference, it should

- include the identical varieties of stains going on in a query photograph

- have photograph history and histological components

stained by distinctive chemical dyes truely provided in any other case, misguided, or even irrelevant, quantities are estimated on this off-line technique, in the end affecting universal normalization performance.

The on line manner normalizes colors in a question photo I.

For every query image, the imaging illuminant and stain spectra are expected and coupled to reference quantities generated via the off-line method. due to the fact aspect data on question photographs training, such as information about imaging tool, is hardly ever guaranteed for big photograph sets, This work focuses on an operational scenario where no facet data on a query picture, other than expertise approximately the stain kind, is available to the device.

As proven in Fig. 2, the complex normalization pipeline is formed through modules, one to normalize imaging illuminant and the alternative to get rid of spectral variation in stains. It ought to be referred to that, PSD of imaging mild influences photo shade linearly within the linear RGB shade space, at the same time as image colour varies following an exponential function with respect to stain spectra. hence it's far important to become aware of the 2 unbiased factors, and advise linear and nonlinear algorithms to normalize coloration version due to the 2 factors for my part. except, as illuminant version and stain variation are impartial and may be addressed one by one, if colour version is thought to be delivered with the aid of one purpose, an photo may be processed with the aid of the corresponding module only.

1) Illuminant Normalization Module: Illuminant variation, similar to inconsistency in biopsy imaging on this work, introduces coloration bias in pictures. To cast off the coloration bias, $\mathrm{E}()$ of a query picture have to be anticipated and matched to a trendy Es(), that's both predicted from a reference photograph or defined by a predetermined amount which include the CIE illuminant D65 [26].however, estimation of $\mathrm{E}()$ without delay from an image is notably tough because of the indispensable impact of a camera. based at the idea of metamer [27], we deduce an equivalent depth matching algorithm to reap illuminant normalization. that is defined in set of rules 1 photograph intensities in clean regions need to be expected for

Illuminant normalization. though most histo-pathology pictures have blank regions, to make sure gadget robustness, we advise the use of a threshold thb (e.g. thb $=2$ hundred for eight bit information) to become aware of photos without clean areas. In particular, if it's miles smaller than thb, it is in all likelihood that the picture does not comprise any blank vicinity. For this type of case, our set of rules will return the photo with a notification to operators/pathologists for further analysis. The whole illuminant normalization module is defined in algorithm 1

2) Spectral Normalization Module :
If a fixed of biopsy samples stained with the aid of the equal types of chemical dyes are imaged the use of one scanner, color variation in pics are specially due to war of words in biopsy staining. even though stains have their personal diagnostic colorations, which includes hematoxylin typically appearing in blue while eosin in pink visually, due to inconsistency in stain manufacture, stain concentration, or garage situation, absorption spectra of stains, may also range, resulting color version in photographs. therefore earlier than quantitative evaluation, color version due to inconsistent stain spectra must be removed. Whilst normalizing spectral variation in stains, care must be taken to keep histological statistics. though shade version amongst snap shots is due to different factors, inside one photograph, those element are consistent and histological facts is conveyed by means of colors As numerous colorations in an photograph are generated by different combinations of stains, histological facts is surely conveyed via stain depths at each pixel, which is denoted with the aid of $\mathrm{D}(\mathrm{p})$. To normalize inconsistent stain spectra $\mathrm{Mi}()$ while to maintain stain proportion $\mathrm{D}(\mathrm{p})$ unchanged, we concatenate a NMF based totally spectral estimation and spectral matching. To simplify the hassle, on this phase, we anticipate images are generated under preferred imaging situation. in any other case, the proposed illuminant normalization could be applied first.

3) NMF-based Spectral Estimation:

For a biopsy pattern stained via $\mathrm{N}$ forms of stains and imaged through ok sensors, we've got $\mathrm{k}$ equations for its corresponding his to-pathology photo. Then a ok-by using-N spectrum matrix $\mathrm{M}$ is fashioned through using $\mathrm{Mi}()$ because the ith row. subsequently, an detail mij in a spectrum matrix $\mathrm{M}$ represents the jth stains absorption spectrum around unique wavelength $i$ and spectrum of the jth stain, $M: j$ can be characterized via the jth column of $\mathrm{M}$. as an instance, an RGB-layout HE stained image has a three-by using-2 spectrum matrix, whose first column corresponds to spectrum vector of hemotoxylin and the second one column to eosin.

The general spectral normalization module on the basis of NMF-primarily based spectral estimation is described in set of algorithm 3.

\subsubsection{Algorithm Used}

1. Illuminant Normalization

- Input data : Linear RGB format query image I,

reference image Is

- Output Data : Normalized query image I

- $\quad$ For (each channel i, i= 1,2,3 ) do

Intensity estimation on reference image

Intensity estimation on input image

- If $\mathrm{Ib}=$ threshold then do Intensity

Matching Else return with a notification

- $\quad$ End

\section{INITIALIZATION USING SW STATISTICS}

- Input Data : Linear RGB format image, Stain type

- Output data : Matrix Min

- $\quad$ RGB to HSV Conversion 
- Using S.W method, the S.W values were identified

- $\quad$ and compute SW- Hue histogram.

- $\quad \mathrm{K}$ - mean clustering

- Converted to RGB and make a matrix Min

- $\quad$ Normalize Min

\section{SPECTRAL NORMALIZATION}

- Input data : Linear RGB format query image I,

- Reference image Is stain type $\mathrm{N}$

- Apply algorithm B to I and Is

- Apply spectral matching to I

\subsection{Feature Extraction}

The feature extraction extracts the functions of significance for image popularity. The characteristic extracted offers the property of the text individual, which may be used for schooling inside the database. The obtained educated characteristic is in comparison with the test sample feature received and categorized as one of the extracted person. Following capabilities are used as enter for the neural community.

- Area (ar)

- $\quad$ Perimeter(pr)
1) Area per cell Apc $=\operatorname{sum}(\mathrm{ar}) / \mathrm{n}$
2) Median of area Med = median (ar)
3) Maximum value of area $\mathrm{Mxv}=\max (\mathrm{ar})$
4) Length $\mathrm{La}=$ find $(\mathrm{ar}==\mathrm{med})$
5) Median of perimeter Medp = median (pr)
6) Perimeter per cell $\mathrm{Ppc}=\operatorname{sum}(\mathrm{pr}) / \mathrm{pr}(\mathrm{la}(1))$
7) Maximum value of perimeter. $\mathrm{Pm}=\max (\mathrm{pr})$
8) Total median Medd = med/medp

\subsection{Back Propagation Neural Network classifier}

Back propagation network (BPN) is a systematic technique for education multi-layer artificial neural network. returned propagation affords a computationally green approach for changing the weights in a feed ahead network with differentiable activation characteristic gadgets to analyze a set of input output examples. The multi-layer feed ahead BPN for category of tumor consists of 3 layers which includes production layer, secret layer and participation layer. the whole range of nodes inside the enter layer is nine representing features extracted from the ROI. The number of nodes in the hidden layer is 5 became determined experimentally as the network produced appropriate effects. The output of BPN is 6 values. The nodes in one layer connect to the nodes in the next layer by using directed verbal exchange links, every with as an related arbitrary burden. The BPNN has 3 kinds of layer together with enter layer, hidden layer and output layer. The hidden layer and output layer having consequences in two weight matrices is w1 and w2. the burden matrices w1 used to attach the enter layer to be hidden layer. the burden matrices w2 is used to connect the hidden layer to the output layer The processing time taken via the network is less than a minute.

\subsection{Calculation Process}

All classification results could have an error rate and on occasion will either fail to identify the normal and abnormal images. It is common to describe this error rate by the terms true and false positive and true and false negative.

Classification Accuracy $(\mathrm{CA})=\mathrm{TP}+\mathrm{TN} /(\mathrm{TP}+\mathrm{TN}+\mathrm{FP}+$ FN)

Sensitivity $=\mathrm{TP} / \mathrm{TP}+\mathrm{FN}$
Specificity $=\mathrm{TN} / \mathrm{TN}+\mathrm{FP}$

Finally Classification result calculated based on the accuracy formula. Usually the Accuracy Measure is used

Accuracy $=$ No of Correctly Classified record $/$ Total Records in the test set

Where

- True Positive TP

- False Negative FN

- True Negative TN

- $\quad$ False Positive FP

- "TP of Benign type1" is all type 1 instances that are classified as type 1

- "TN of Benign type1" is all non- Benign type1 instances that are not classified as Benign type1.

- "FP of Benign type1" is all non- Benign typelinstances that are classified as Benign type1.

- "FN of Benign type1" is all Benign type1 instances that are not classified as Benign type 1.

\section{RESULTS AND DISCUSSION}

Diverse experiments were carried out and the education and the trying out units were decided by means of taking into attention the class accuracies. The facts set became divided into separate information units the training records set and the checking out statistics set. The schooling manner is used to educate the information set and the testing technique is used to affirm the accuracy and effectiveness of the skilled network for the type of mind tumors. The proposed method is tested greater than fifty five mammogram images. These paintings applied by the use of MATLAB R-2014 surroundings. The determine suggests the given input cancerous images used for the image category steps. The input snap shots are categorized by back propagation neural network. those neural networks are skilled using returned propagation technique. The result of lower back Propagation Neural community (BPNN) is calculated through unidentified testing pix. The category results of again Propagation Neural Networks are shown in figures. 


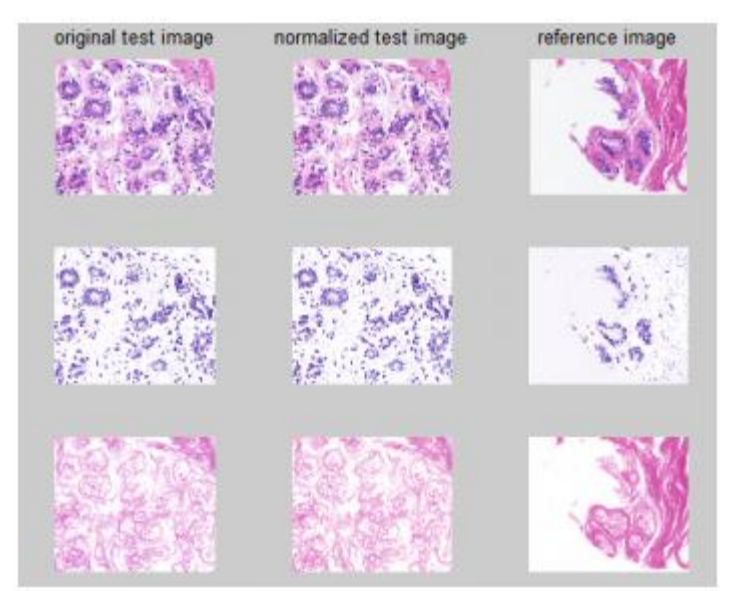

Fig. 3 Result of Color Normalisation scheme

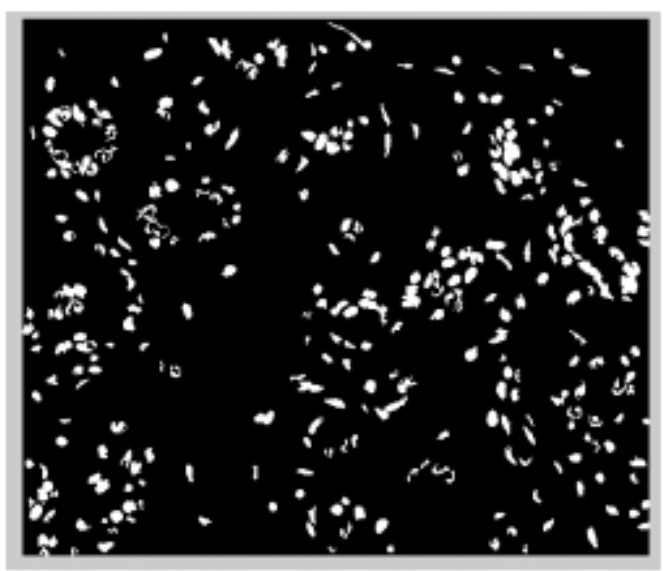

Fig.4. Pre-processing Result :Feature extraction Binarization

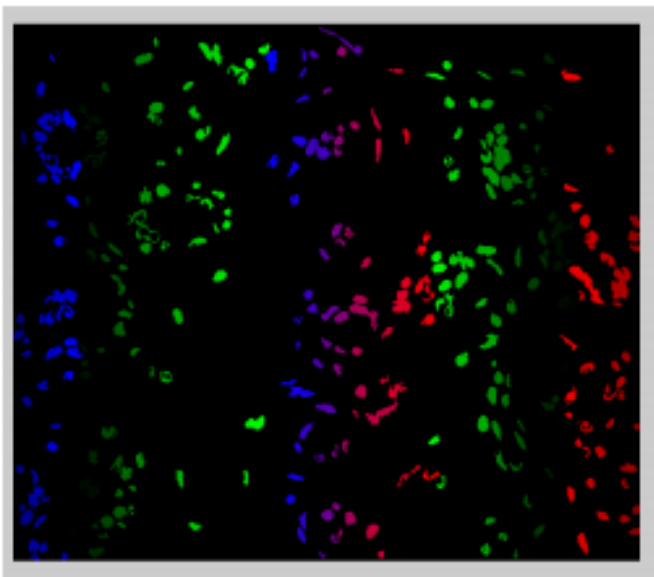

Fig. 5. Pre-processing Result: RGB Conversion

At some stage in training, the following window shown in parent nine opens. This window shows training development and permits to break schooling at any point by using clicking stop education.

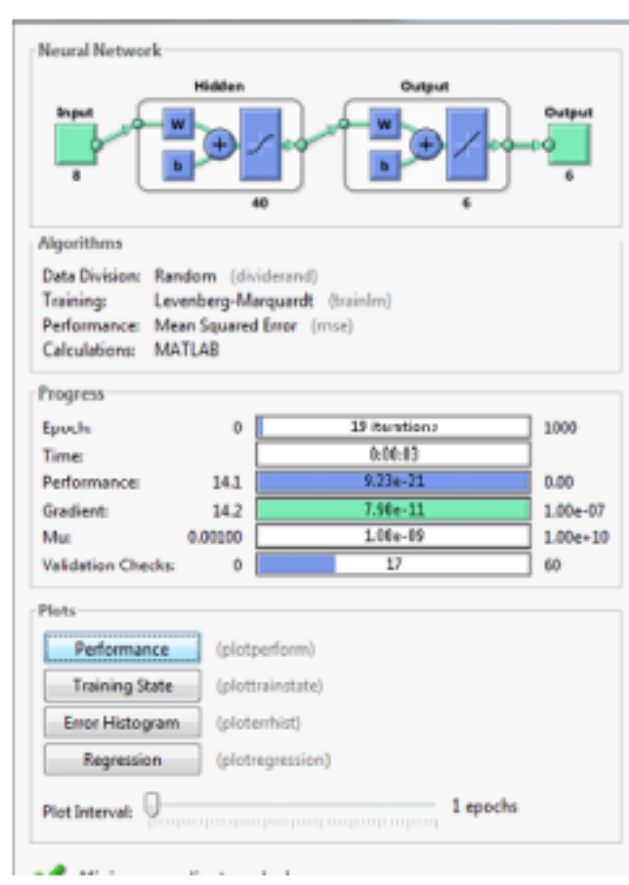

Fig. 6. Neural network Training window

The overall performance result is shown in figure 7. it's miles reasonable because of the subsequent issues:

- The final mean-square error is small

- The test set error and the validation set error has similar characteristics

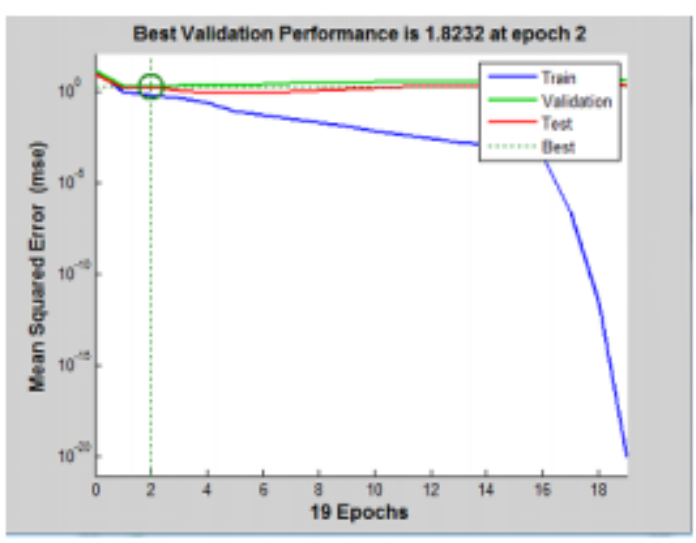

Fig. 7. Performance analysis

The output tracks the objectives very well for education, checking out, and validation, and the R-price is over 0.53476 for the overall reaction. The regression value is proven in figure 11 shade normalization is an crucial step to put off inherent coloration variation in histo pathological snap shots and as a result is useful for analyzing disorder and its progression on huge datasets from one-of-a-kind pathology labs. A capacity hazard of such a technique is that tissue systems offered within the original picture could be altered after normalization. So here behavior experiments to compare the proposed normalization method. the subsequent public histo-pathology picture set are used as experimental data on 
this work. they are decided on in exceptional experiments depending on experimental objectives.

The USA breast cancer cellular dataset [55] was published for an goal of cell segmentation in both benign and malignant cellular photos. The dataset includes cancerous cellular pix reduce from $10 \mathrm{HE}$ stained breast cancer biopsies. All snap shots are scanned within the identical laboratory. as the u.s. dataset store pics in 24- bit non-linear RGB layout, all pics are transformed to the linear RGB domain first [27]. All algorithms in this paper are implemented in Mat lab.

\section{CONCLUSION AND FUTURE ENHANCEMENT}

Breast cancer mobile picture category with feature choice and extraction has been finished in the beyond with restricted achievement. in this research synthetic neural community again propagation approach is applied to categories benign and malignant disorder in mammogram photos. A sturdy and whole coloration normalization technique capable of addressing shade version in histopathology pics is added here as a preprocessing technique. based on an imaging version, the added answer become capable of discover the source of shade variation, and addressed efficiently both illuminant variant and stain version using an depth matching set of rules and a spectral normalization module respectively. The saturation weighted stain estimation technique introduced with the aid of this work limited impacts of achromatic spectra on stain estimation and robustified the estimation system. full-size experimentation on publicly available datasets suggests that the proposed answer outperforms contemporary colour normalization solutions, while retaining histological records. Then lower back propagation set of rules neural network approach was stepped forward to teach and checking out the tumor pics. The proposed BPNN gives $90 \%$ classification accuracy in most cancers image taxonomy. The proposed method achieves outcomes as follows category accuracy $90 \%$ and it's also minimizing the mistake charge as well as increase the classification accuracy. The processing time taken by means of the community is less than a minute. This the proposed technique performs better than the winning works. In future, these paintings can be carried out in massive quantity of datasets.

\section{ACKNOWLEDGMENT}

I thank God almighty for all the blessings received during this endeavour. I would like to offer my deepest gratitude to everybody who helped me directly and indirectly for the successful completion of my work. Last, but not the least, I thank all my friends and my family for the support and encouragement they have given me during the course of my work.

\section{REFERENCES}

[1] Xingyu Li, Student Member, IEEE, and Konstantinos N. Plataniotis, Fellow, IEEE,"A Complete Color Normalization Approach to Histo-pathology Images
Using Color Cues Computed From SaturationWeighted Statistics",IEEE Transactions on Biomedical Engineering

[2] K. Jafari-Khouzani and H. Soltanian-Zadeh, Multiwavelet grading ofpathological images of prostate, IEEE Trans. Biomed. Eng., vol. 50, no. 6, pp. 697 704, Jun. 2003.

[3] A. Basavanhally et al., Computerized image-based detection and grading of lymphocytic infiltration in her2+ breast cancer histopathology, IEEE Trans. Biomed. Eng., vol. 57, no. 3, pp. 642 653, Mar. 2010

[4] K. Nguyen et al., Structure and context in prostatic gland segmentation and classification, in Proc. MICCAI, 2012.

[5] L. Gorelick et al., Prostate histopathology: Learning tissue component histograms for cancer detection and classification, IEEE Trans. Med. Imag., vol. 32, no. 10, pp. 1804 1818, Oct. 2013.

[6] M. Veta et al., Breast cancer histopathology image analysis: A revier, IEEE Trans. Biomed. Eng., vol. 61, no. 5, pp. 1400 1411, May 2014.

[7] M.N. Gurcan et al., Histopathological image analysis: a review, IEEE Rev. Biomed. Eng., vol. 2, pp. 147 171, 2009.

[8] M. Macenko et al., A method for normalizing histology slides for quantitative analysis, in Proc. ISBI, 2009.

[9] A. Tabesh et al., Multifeature prostate cancer diagnosis and gleason grading of histological images, IEEE Trans. Med. Imag., vol. 26, no. 10, pp. 1366 1378, Oct. 2007.

[10] S. Kothari et al., Automatic batch-invariant color segmentation of histological cancer images, in Proc. ISBI, 2011.

[11] Y. Wang et al., A color-based approach for automated segmentation in tumor tissue classification, in Proc. EMBC, 2007.

[12] D. Magee et al., Color normalization in digital histopathology images, in Proc. MICCAI OPTMHisE, 2009.

[13] A.M. Khan et al., A non-linear mapping approach to stain normalization in digital histopathology images using image-specic color deconvolution, IEEE Trans. Biomed. Eng., vol. 61, no. 6, pp. 1729 1738, Jun. 2014.

[14] S. Tani et al., Color standardization method and system for whole slide imaging based on spectral sensing, Analytical Cellular Pathology, vol. 35, no. 2, pp. 107 115, 2012.

[15] A. Basavanhally and A. Madabhushi, Em-based segmentatio-driven color standardization of digitized histopathology, in Proc. SPIE Medical Imaging 2013: Digital Pathology, 2013, vol. 8676 